\title{
M1A/M1B ESOPHAGEAL CARCINOMA: CLINICAL RELEVANCE
}

Neil A. Christie, MDa

Thomas W. Rice, MD ${ }^{a}$

Malcolm M. DeCamp, MD

John R. Goldblum, MD

David J. Adelstein, MD $^{\mathrm{c}}$

Gregory Zuccaro, Jr, MD

Lisa A. Rybicki, MS

Eugene H. Blackstone, MD ${ }^{\mathrm{a}, \mathrm{e}}$
Objective: The 1997 staging system for esophageal carcinoma subdivides distant metastatic disease (M1) into M1a (nonregional lymph node metastases) and M1b (other metastases). This study evaluates the relevance of this classification. Methods: One hundred forty patients were identified with M1 disease, 36 (26\%) M1a and 104 (74\%) M1b. The histologic type was adenocarcinoma in $118(84 \%)$, squamous cell in 18 $(13 \%)$, and adenosquamous in $4(3 \%)$, with a similar distribution for M1a and M1b $(P=.3)$. Forty-five underwent surgery, $28(78 \%)$ with M1a disease and $17(16 \%)$ with M1b disease $(P<.001)$. Chemotherapy and/or radiation therapy was given to $33(73 \%)$ surgical patients and 63 $(66 \%)$ nonsurgical patients $(P=.4), 28(78 \%)$ with M1a disease and 68 $(66 \%)$ with M1b disease $(P=.17)$. Results: Median and 5-year survivals were 11 months and $6 \%$ in patients with M1a disease and 5 months and $2 \%$ in those with M1b disease $(P=.001)$. Surgery provided no advantage in M1b $(P=.6)$ or M1a disease $(P=.2)$. Multivariable analysis demonstrated that patients with M1b disease had 1.8 times the mortality risk of those with M1a disease (CI 1.2-2.7, $P=.004$ ), and patients without chemotherapy and/or radiotherapy had 2.2 times the mortality risk of those with chemotherapy and/or radiotherapy (CI 1.5-3.2, $P$ < $.001)$. Despite the prevalence of surgery in patients with M1a disease, the analysis suggests that M1a and use of chemotherapy and/or radiotherapy, rather than surgery, account for the small, clinically unimportant differences in survival. Conclusions: We conclude that (1) although there are statistically significant survival differences between M1a and M1b disease, these differences are not clinically important; (2) chemotherapy and/or radiotherapy is associated with a modest survival benefit; and (3) surgery offers no survival advantage. (J Thorac Cardiovasc Surg 1999;118:900-7)
$\mathrm{R}^{\mathrm{s}}$ ecent revision of the staging system for esophageal carcinoma subdivides distant metastatic carcinoma (M1) into M1a (distant, nonregional lymph node metastases) and M1b (other distant metastases). ${ }^{1}$ M1a disease

From the Center for Swallowing and Esophageal Disorders, Department of Thoracic and Cardiovascular Surgery, ${ }^{\text {a Department }}$ of Anatomic Pathology, ${ }^{b}$ Department of Hematology and Medical Oncology, ${ }^{\mathrm{c}}$ Department of Gastroenterology, ${ }^{\mathrm{d}}$ and Department of Biostatistics and Epidemiology, ${ }^{\mathrm{e}}$ The Cleveland Clinic Foundation, Cleveland, Ohio.

Read at the Seventy-ninth Annual Meeting of The American Association for Thoracic Surgery, New Orleans, La, April 18-21, 1999.

Received for publication April 22, 1999; revisions requested July 8, 1999; revisions received July 21, 1999; accepted for publication Aug 13, 1999.

Address for reprints: Thomas W. Rice, MD, The Cleveland Clinic Foundation, 9500 Euclid Ave, Desk F25, Cleveland, OH 44195 (E-mail: ricet@ccf.org).

Copyright (C) 1999 by Mosby, Inc.

0022-5223/99 $\$ 8.00+0 \quad \mathbf{1 2 / 6 / 1 0 2 0 9 2}$ is further classified by tumor location; M1a tumors of the upper thoracic esophagus metastasize to cervical nodes and M1a tumors of the lower thoracic esophagus metastasize to celiac lymph nodes (Table I).

This study evaluates the clinical relevance and treatment implications of this new classification of distant metastatic disease.

\section{Patients and methods}

From our prospective surgical database (patients undergoing resection of esophageal carcinoma at The Cleveland Clinic Foundation between January 1, 1983, and December $31,1996)$ and our tumor registry (patients with esophageal carcinoma treated at The Cleveland Clinic Foundation between January 1, 1986, through December 31, 1996), 140 patients with M1 disease were identified. Patient age at presentation, sex, race, prior surgery, prior esophageal or gastric surgery, use of endoscopic esophageal ultrasound (EUS), histologic cell type, histologic differentiation, presence of 
Table I. Staging definitions

\begin{tabular}{|c|c|}
\hline Site & Definition \\
\hline \multicolumn{2}{|l|}{ Primary } \\
\hline Upper thoracic esophagus & Extends from the thoracic inlet to the tracheal carina, approximately 18 to $24 \mathrm{~cm}$ from the incisor teeth \\
\hline Mid thoracic esophagus & Extends from the tracheal carina to the inferior pulmonary vein, approximately 24 to $32 \mathrm{~cm}$ from the incisor teeth \\
\hline Lower thoracic esophagus & $\begin{array}{l}\text { Extends from the inferior pulmonary vein to the esophagogastric junction including the intra-abdominal esophagus, } \\
\text { approximately } 32 \text { to } 40 \mathrm{~cm} \text { from the incisor teeth }\end{array}$ \\
\hline \multicolumn{2}{|l|}{ Distant metastases } \\
\hline \multirow[t]{2}{*}{ M1a } & Upper thoracic esophagus metastatic to cervical lymph nodes \\
\hline & Lower thoracic esophagus metastatic to celiac lymph nodes \\
\hline \multirow[t]{3}{*}{ M1b } & Upper thoracic esophagus metastatic to noncervical nonregional lymph nodes or other distant sites \\
\hline & Mid thoracic esophagus metastatic to either nonregional lymph nodes or other distant sites \\
\hline & Lower thoracic esophagus metastatic to nonceliac nonregional lymph nodes or other distant sites \\
\hline
\end{tabular}

Table II. Patient and carcinoma characteristics

\begin{tabular}{|c|c|c|c|c|c|c|c|}
\hline \multirow[b]{2}{*}{ Variable } & \multicolumn{2}{|c|}{ All patients } & \multicolumn{2}{|c|}{ M1a } & \multicolumn{2}{|c|}{$M 1 b$} & \multirow[b]{2}{*}{$\mathrm{P}$} \\
\hline & $N$ & $\%$ of 140 & $N$ & $\%$ of 36 & $N$ & $\%$ of 104 & \\
\hline Male sex & 115 & 82 & 34 & 94 & 81 & 78 & .02 \\
\hline EUS & 57 & 41 & 20 & 56 & 37 & 36 & .04 \\
\hline Cell type & & & & & & & .3 \\
\hline Adenocarcinoma & 118 & 84 & 33 & 92 & 85 & 82 & \\
\hline Squamous cell & 18 & 13 & 2 & 6 & 16 & 15 & \\
\hline Adenosquamous & 4 & 3 & 1 & 3 & 3 & 3 & \\
\hline Differentiation & & & & & & & .6 \\
\hline Poor & 82 & 59 & 19 & 53 & 63 & 61 & \\
\hline Moderate-poor & 11 & 8 & 4 & 11 & 7 & 7 & \\
\hline Moderate & 35 & 25 & 9 & 25 & 26 & 25 & \\
\hline Moderate-well & 6 & 4 & 3 & 8 & 3 & 3 & \\
\hline Well & 6 & 4 & 1 & 3 & 5 & 5 & \\
\hline Barrett's mucosa & 32 & 23 & 9 & 25 & 23 & 22 & .7 \\
\hline Primary site & & & & & & & .2 \\
\hline Upper & 2 & 1 & 0 & 0 & 2 & 2 & \\
\hline Mid & 7 & 5 & 0 & 0 & 7 & 7 & \\
\hline Lower & 131 & 94 & 36 & 100 & 95 & 91 & \\
\hline
\end{tabular}

EUS, Esophageal ultrasound.

Barrett's mucosa, primary tumor site, and distant metastatic status (M1a or M1b) were recorded. Therapies recorded included surgery, chemotherapy and/or radiotherapy, and local palliative therapy (stent or endoscopic lesion ablation).

Statistical methods. Descriptive statistics are summarized as the mean and standard deviation for continuous variables and as frequencies and percentages for categoric variables. Continuous variables were compared between patients with M1a and M1b tumors by means of the $t$ test; categoric variables were compared by means of the $\chi^{2}$ test. The Kaplan-Meier method was used to estimate survival and the log-rank test was used to compare survival between patients with M1a and M1b disease. Cox proportional hazards analysis was used to identify univariable and multivariable correlates of survival. Two models of survival were developed. In the first, the influence on survival of M1a versus M1b was simply adjusted for the confounding of surgery. In the second, stepwise analysis of the variables described above (and in Table VI) were considered, except for cell type, because of low prevalence of
Table III. Site of metastatic disease for 104 patients with $M 1 b$ disease

\begin{tabular}{lrc}
\hline Site & $N$ & \% of 104 \\
\hline Single & & \\
Liver & 36 & 35 \\
Lung & 16 & 15 \\
Bone & 13 & 13 \\
Adrenal & 3 & 3 \\
Brain & 1 & 1 \\
Other & 30 & 29 \\
Multiple & 5 & 5 \\
Lung and liver & 2 & 2 \\
Liver and bone & 2 & 2 \\
Lung, liver, and bone & 1 & 1 \\
\hline
\end{tabular}

adenosquamous cancers. In addition, to assess the possible influence of increased experience across time, the date of diagnosis was evaluated. Variables were retained in the model for $P<.05$. 
Table IV. Chemotherapy and radiotherapy

\begin{tabular}{|c|c|c|c|c|c|c|c|c|}
\hline \multirow{2}{*}{$\frac{\text { Patients }}{\text { Surgical }}$} & \multirow[b]{2}{*}{ Therapy } & \multicolumn{2}{|c|}{ All patients } & \multicolumn{2}{|c|}{ M1a } & \multicolumn{2}{|c|}{$M 1 b$} & \multirow{2}{*}{$\frac{\mathrm{P} *}{.5}$} \\
\hline & & $N$ & $\%$ of 45 & $n$ & $\%$ of 28 & $n$ & $\%$ of 17 & \\
\hline & Chemo/RT & 13 & 29 & 9 & 32 & 4 & 24 & \\
\hline & Chemo only & 2 & 4 & 2 & 7 & 0 & 0 & \\
\hline & RT only & 18 & 40 & 11 & 39 & 7 & 41 & \\
\hline & None & 12 & 27 & 6 & 21 & 6 & 35 & \\
\hline \multirow[t]{5}{*}{ Nonsurgical } & Therapy & $N$ & $\%$ of 95 & $n$ & $\%$ of 8 & $n$ & $\%$ of 87 & .13 \\
\hline & Chemo/RT & 12 & 13 & 3 & 38 & 9 & 10 & \\
\hline & Chemo only & 20 & 21 & 2 & 25 & 18 & 21 & \\
\hline & RT only & 31 & 33 & 1 & 12 & 30 & 34 & \\
\hline & None & 32 & 34 & 2 & 25 & 30 & 34 & \\
\hline \multirow[t]{5}{*}{ All patients } & Therapy & $N$ & $\%$ of 140 & $n$ & $\%$ of 36 & $n$ & $\%$ of 104 & .04 \\
\hline & Chemo/RT & 25 & 18 & 12 & 33 & 13 & 12 & \\
\hline & Chemo only & 22 & 16 & 4 & 11 & 18 & 17 & \\
\hline & RT only & 49 & 35 & 12 & 33 & 37 & 36 & \\
\hline & None & 44 & 31 & 8 & 22 & 36 & 35 & \\
\hline
\end{tabular}

Chemo, Chemotherapy; $R T$, radiotherapy.

${ }^{*} P$ for difference in pattern of therapies between M1a and M1b groups based on $\chi^{2}$ test.

Table V. Type of local therapy

\begin{tabular}{|c|c|c|c|c|c|c|}
\hline \multirow[b]{2}{*}{ Type } & \multicolumn{2}{|c|}{ All patients } & \multicolumn{2}{|c|}{ M1a } & \multicolumn{2}{|r|}{$M 1 b$} \\
\hline & $n$ & $\%$ of 140 & $n$ & $\%$ of 36 & $n$ & $\%$ of 104 \\
\hline Stent & 9 & 6 & 1 & 3 & 8 & 8 \\
\hline $\begin{array}{c}\text { Endoscopic } \\
\text { ablation }\end{array}$ & 35 & 25 & 2 & 6 & 33 & 32 \\
\hline Both & 11 & 8 & 2 & 6 & 9 & 9 \\
\hline None & 85 & 61 & 31 & 86 & 54 & 52 \\
\hline
\end{tabular}

$P=.003$ for difference in types of local therapy between M1a and M1b $\left(\chi^{2}\right)$.

\section{Results}

Patients. A total of 140 patients with esophageal carcinoma metastatic to distant sites were identified: 36 patients with M1a disease and 104 with M1b. Mean age of the group was $62 \pm 10(\mathrm{SD})$ years (range 32-84 years) and was not different between patients with M1a disease (mean $61 \pm 10$ years, range $44-83$ years) and those with M1b disease (mean $62 \pm 10$ years, range 3284 years) $(P=.6)$. Descriptive characteristics are listed in Table II. Sites of distant metastases for the M1b group are listed in Table III. Multiple sites were rare, occurring in only 5 patients, all of whom received nonoperative therapy.

Treatment. Forty-five patients were treated primarily with surgery. Patients with M1a disease $(\mathrm{n}=28)$ were more likely to undergo surgery than patients with M1b disease (n = 17) $(78 \%$ vs $16 \%, P<.001)$. Of the 17 patients with M1b disease who were treated surgi- cally, 9 underwent palliative resections, and 8 were committed to resection before the M1b status was determined. Chemotherapy and/or radiotherapy was administered to 33 surgical patients, 22 with M1a disease (79\%) and 11 with M1b disease (65\%) (Table IV). Nine of these received neoadjuvant therapy, $7 \mathrm{M} 1 \mathrm{a}$ and $2 \mathrm{M} 1 \mathrm{~b}$. In addition, 24 received postoperative adjuvant therapy only, $15 \mathrm{M} 1 \mathrm{a}$ and $9 \mathrm{M} 1 \mathrm{~b}$.

Therapies for the 95 patients who did not undergo surgery are listed in Table IV. Among these patients, combined chemotherapy and radiotherapy was more common in those with M1a disease $(3 / 8,38 \%)$ than in those with M1b disease $(9 / 87,10 \%)(P=.03)$. It is this specific difference in the nonsurgical group that accounts for the overall difference in the pattern of therapy between M1a and M1b ( $P=.04$, Table IV). Local therapy was administered to 55 patients (Table V).

Survival. Median length of follow-up (presentation until death or last follow-up) was 6.4 months: 10.9 months (range 1.3-60 months) in the M1a group and 5.1 months (range 0.2-69 months) in the M1b group. Five patients (3 M1a, 2 M1b) are currently alive. Median and 5-year Kaplan-Meier survival estimates are 11 months (95\% CL 9-16 months) and 6\% (95\% CL $0 \%-14 \%$ ) for patients with M1a disease and 5 months (95\% CL 4-7 months) and 2\% (95\% CL 0\%$5 \%)$ for those with M1b disease $(P=.001)$ (Fig 1). For M1a, surgical therapy afforded no clinically relevant survival benefits $(P=.2)$, although the curves diverge at 12 months (Fig 2). Survival was similar for 


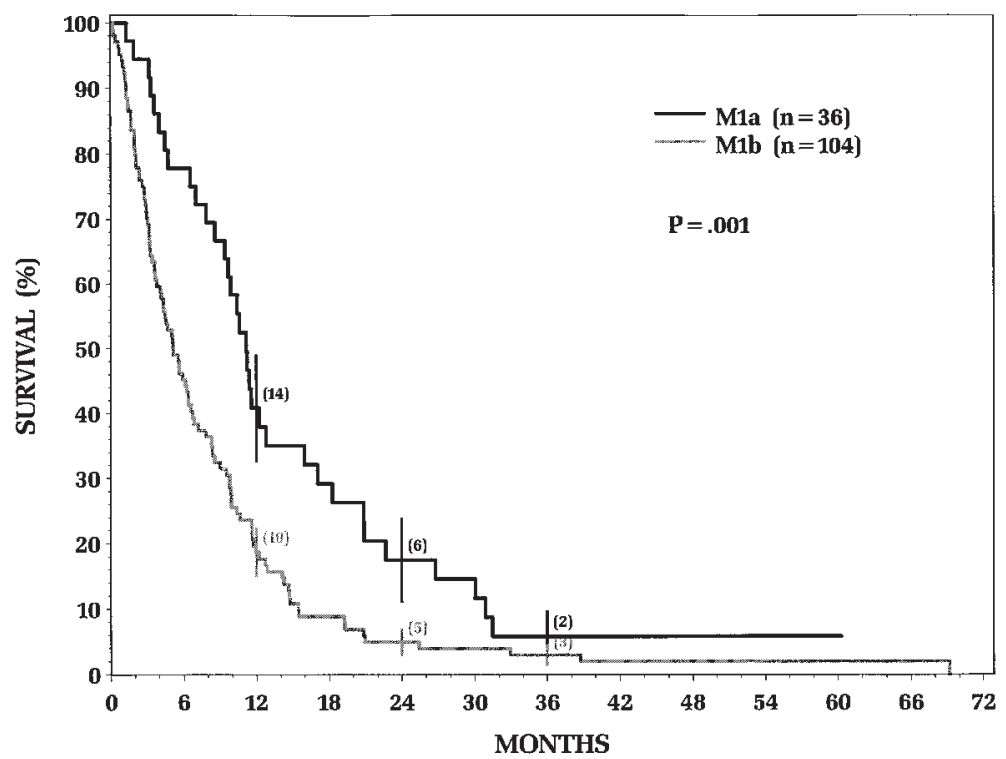

Fig 1. Survival from presentation to death or last follow-up in patients with M1a (solid line) or M1b (light line) disease. The vertical bars represent 1 standard error of the survival estimates. The numbers in parentheses represent the number traced at 12,24 , and 36 months.

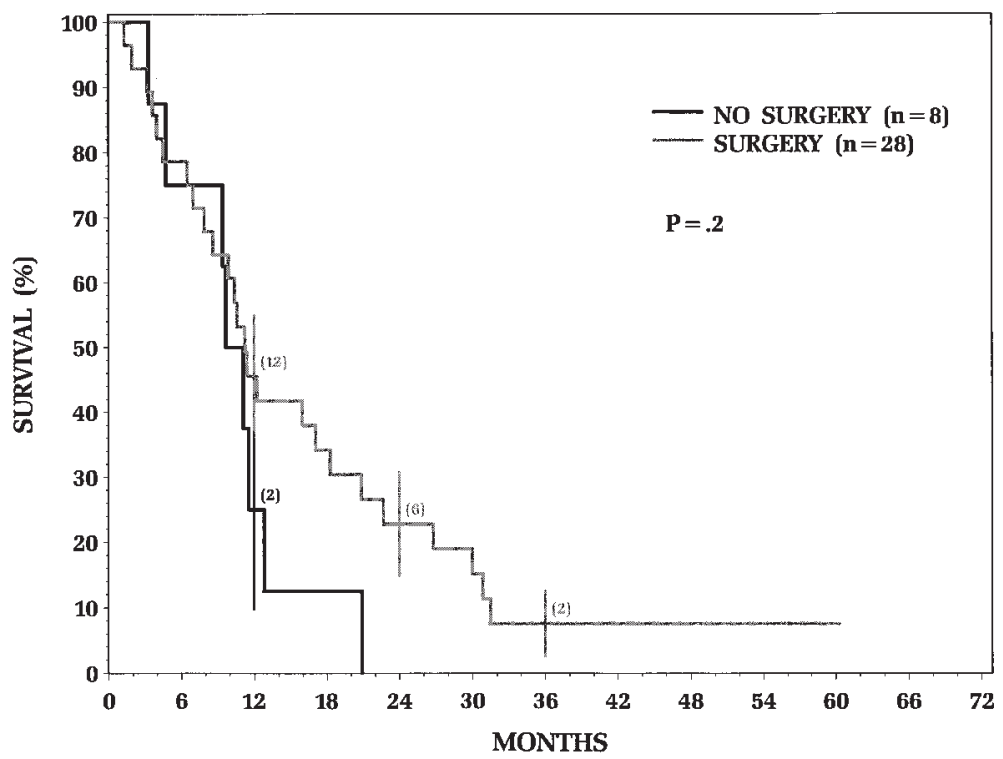

Fig 2. Survival from presentation to death or last follow-up in patients with M1a disease stratified by surgical (solid line) versus nonsurgical (light line) therapy. The format is as in Fig 1.

surgical and nonsurgical therapy in the M1b group $(P=.6)$.

For patients receiving surgical therapy, pathologic $\mathrm{T}$ status (pT) was not predictive of survival $(P=.3)$. Only 3 patients did not have regional lymph node metastases (N0), and therefore survival analysis was precluded. For the 42 patients with regional lymph node metas- tases (N1), the total number of positive nodes and percent of nodes positive were not predictive of survival ( $P$ $=.7$ and .4 , respectively).

Univariable correlates of survival are listed in Table VI. Two multivariable models are presented in Table VII. In both, patients with M1b disease were at higher risk than those with M1a disease. In the first model, 
Table VI. Univariable correlates of survival (Cox

\begin{tabular}{|c|c|c|c|}
\hline Variable/response & $H R$ & $95 \% C I$ & $\mathrm{P}$ \\
\hline \multicolumn{4}{|l|}{ Sex } \\
\hline Female/male & 1.2 & $0.8-1.8$ & .5 \\
\hline \multicolumn{4}{|l|}{ Age at presentation } \\
\hline Per 10-year increase & 1.2 & $0.98-1.4$ & .08 \\
\hline \multicolumn{4}{|l|}{ Race } \\
\hline White/other & 0.8 & $0.4-1.6$ & 6 \\
\hline \multicolumn{4}{|l|}{ Prior surgery } \\
\hline Yes/no & 0.9 & $0.6-1.3$ & 6 \\
\hline \multicolumn{4}{|c|}{ Prior esophageal/gastric surgery } \\
\hline Yes/no & 0.8 & $0.3-2.0$ & .7 \\
\hline \multicolumn{4}{|l|}{ Endoscopic ultrasound } \\
\hline Yes/no & 0.9 & $0.6-1.2$ & .5 \\
\hline \multicolumn{4}{|l|}{ Cell type } \\
\hline $\mathrm{SQ} / \mathrm{AD}$ & 1.0 & $0.6-1.7$ & .98 \\
\hline $\mathrm{AS} / \mathrm{AD}$ & 3.3 & $1.2-9.1$ & .02 \\
\hline \multicolumn{4}{|l|}{ Barrett's mucosa } \\
\hline Yes/no & 0.9 & $0.6-1.4$ & .8 \\
\hline \multicolumn{4}{|l|}{ Metastatic disease group } \\
\hline M1b/M1a & 1.9 & $1.3-2.8$ & .002 \\
\hline \multicolumn{4}{|l|}{ Type of therapy } \\
\hline Nonsurgical/surgical & 1.7 & $1.2-2.4$ & .007 \\
\hline \multicolumn{4}{|c|}{$\begin{array}{l}\text { Any chemotherapy and/or } \\
\text { radiotherapy }\end{array}$} \\
\hline No/yes & 2.3 & $1.6-3.4$ & $<.001$ \\
\hline \multicolumn{4}{|l|}{ Any chemotherapy } \\
\hline No/yes & 2.0 & $1.4-2.9$ & $<.001$ \\
\hline \multicolumn{4}{|l|}{ Any radiation therapy } \\
\hline No/yes & 1.4 & $0.96-1.9$ & .08 \\
\hline \multicolumn{4}{|c|}{$\begin{array}{l}\text { Local therapy (stent, endoscopic } \\
\text { ablation, both) }\end{array}$} \\
\hline Yes/no & 1.3 & $0.9-1.8$ & .17 \\
\hline \multicolumn{4}{|l|}{ Year of presentation } \\
\hline Per 1-year increase & 1.02 & $0.97-1.07$ & .5 \\
\hline
\end{tabular}

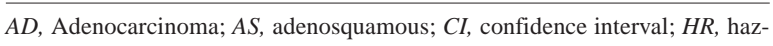
ard ratio; $S Q$, squamous cell. Note: Hazard ratios > 1 indicate a higher risk of mortality.

surgical therapy provided a survival benefit; however, surgical therapy and M1a are confounded. A second model shows that any chemotherapy and/or radiotherapy offers a survival benefit regardless of M1 substage or surgical therapy (Fig 3).

\section{Discussion}

Limitations. This study is a single-institution report of a population that by definition is heterogeneous. All patients undergoing surgery had a pathologic determination of stage. Those patients not receiving surgery had a combination of clinical and pathologic staging. Treatment of this varied group of patients was heterogeneous and included palliation alone, surgery, chemotherapy and/or radiotherapy, or combinations of these and surgery. This study did not include 4 patients
Table VII. Risk factors for death

\begin{tabular}{|c|c|c|c|}
\hline Variable/response & $H R$ & $95 \% C I$ & $\mathrm{P}$ \\
\hline \multicolumn{4}{|l|}{ Model 1} \\
\hline \multicolumn{4}{|l|}{ Metastatic disease group } \\
\hline M1b/M1a & 1.7 & $1.01-2.7$ & .04 \\
\hline \multicolumn{4}{|l|}{ Type of therapy } \\
\hline Nonsurgical/surgical & 1.2 & $0.8-2.0$ & .3 \\
\hline \multicolumn{4}{|l|}{ Model $2^{*}$} \\
\hline \multicolumn{4}{|l|}{ Metastatic disease group } \\
\hline M1b/M1a & 1.8 & $1.2-2.7$ & .004 \\
\hline \multicolumn{4}{|c|}{ Any chemotherapy and/or radiotherapy } \\
\hline No/yes & 2.2 & $1.5-3.2$ & $<.001$ \\
\hline
\end{tabular}

whose condition was clinically staged as M1a and downstaged to M0 with chemotherapy and radiotherapy and who then underwent surgery (Table VIII). Two are alive without disease with a $50 \%$ survival at 13 months and beyond (95\% CI 9\%-91\%). Despite these limitations, useful conclusions can be drawn.

M1 subsets. Identification of nonregional lymph node metastases as a separate staging subgroup has surgical appeal. The progression of metastatic lymph node disease to a more distant site, still within surgical fields, has prompted many to consider radical multiple field lymphadenectomy. However, its similarity to distant disease (M1) was suggested by a comparable survival $(5.5 \%$ at 5 years) in patients with both nonregional lymph node metastases and other distant metastases. ${ }^{2}$ The presence of distant metastatic disease, be it M1a or M1b, overwhelms the importance of $\mathrm{T}$ and $\mathrm{N}$ and necessitates a separate stage group. For carcinomas of the lower thoracic esophagus with celiac lymph node metastases, the present study identifies a somewhat improved survival for these patients (M1a) compared with those with other distant metastases (M1b). However, this doubling of median survival (5 months vs 11 months) and tripling of 5 -year survival ( $2 \%$ vs $6 \%$ ) is clinically unimportant because the increased survival is consumed by the treatment and recovery of the patient. This division of M1 staging has only anatomic merit and statistical significance. To be of clinical significance, survival must extend beyond the treatment and recovery phases.

Therapeutic implications. The present study has therapeutic implications. There is a bias among surgeons to consider nonregional lymph node metastases of esophageal carcinoma as an extension of regional lymph node metastases (N1). Previously there have been calls for classifying this entity as N2 disease, thus implying that nonregional lymph node metastases are 


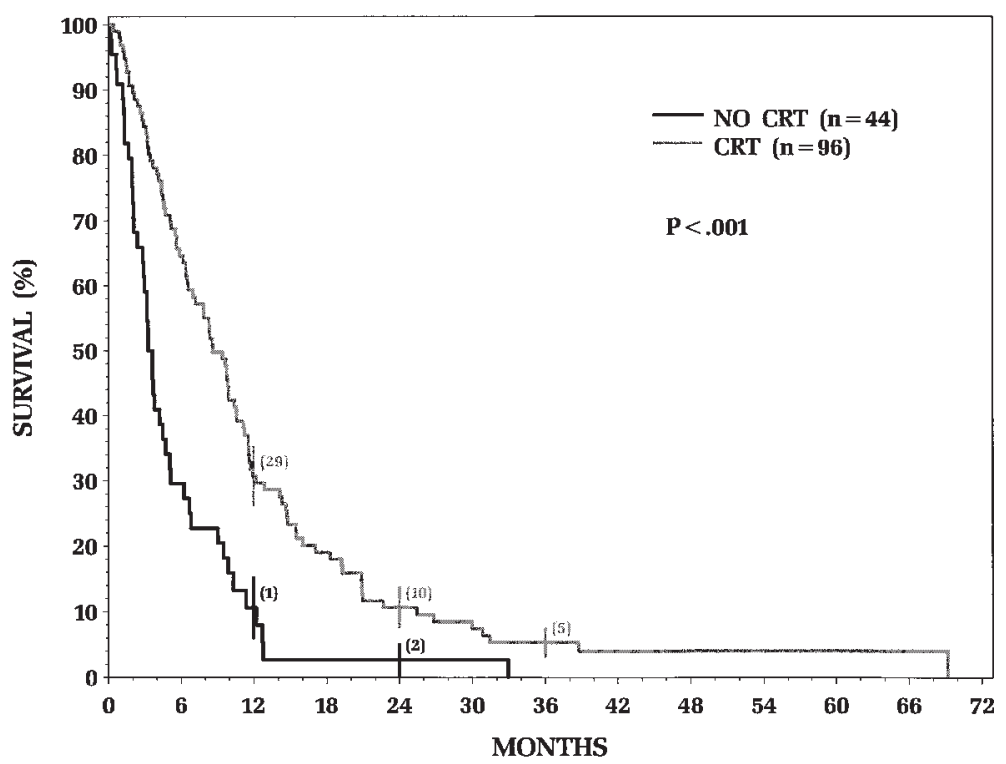

Fig 3. Survival from presentation to death or last follow-up in patients stratified without (solid line) or with (light line) chemotherapy and/or radiotherapy. The format is as in Fig 1.

Table VIII. Mla patients whose conditions were downstaged to MO

\begin{tabular}{lccccr}
\hline Patient & Cell type & Pretreatment stage & Pathologic stage & Status & Follow-up (months) \\
\hline 1 & AD & T3 N1 M1a & T0 N0 M0 & Alive, NED & 79 \\
2 & AD & T3 N1 M1a & T3 N1 M0 & Dead, M1b & 4 \\
3 & AD & T3 N1 M1a & T1 N0 M0 & Dead, NED & 13 \\
4 & SQ & T3 N1 M1a & T0 N0 M0 & Alive, NED & 25 \\
\hline
\end{tabular}

$A D$, Adenocarcinoma; $N E D$, no evidence of disease; $S Q$, squamous cell carcinoma.

resectable. ${ }^{3,4}$ This is an important controversy, since $20 \%$ or more of surgical candidates with carcinoma of the lower thoracic esophagus or carcinoma of the esophagogastric junction will have celiac metastases. In these patients, a 5 -year survival of $15 \%$ has been reported with resection and radical lymphadenectomy. ${ }^{3}$ In the present study, there is minimal difference in survival of patients with M1a disease treated surgically versus those treated nonsurgically. However, long-term survival is seen only in the rare patient undergoing surgical therapy ( $8 \% \pm 5 \%$ at 5 years). This suggests that there are occasional patients with M1a disease who will have long-term survival after resection. Presently these patients cannot be identified before resection and their survival may be independent of surgery. Are we justified in subjecting the vast majority of patients to ineffective morbid therapy for the benefit of the occasional patient? Chemotherapy and/or radiotherapy provides a survival advantage for these patients regardless of M1 subgroup and should be considered the standard against which further therapies should be measured.

\section{Conclusions}

Metastases to nonregional lymph nodes and distant sites are ominous findings in patients with esophageal carcinoma. This study confirms the anatomic and statistical significance of this subdivision of metastatic disease. However, this staging modification does not provide prognosticators within these groups that identify improved survival and that can be used to direct therapy. If a clinically important survival advantage for patients with M1a disease is to be obtained, improvement of nonsurgical therapy is required. Surgery should be considered as an adjunct to this therapy and should be used only in a protocol setting. Otherwise, surgery is the most aggressive form of palliation but not the most effective.

We thank Diane Baisden for data collection and patient follow-up and Lucinda Mitchin for manuscript preparation. 


\section{REFERENCES}

1. Fleming ID, Cooper JS, Henson DE, et al. AJCC cancer staging manual. Esophagus, 5th ed. Philadelphia: Lippincott-Raven; 1997. p. 65-9.

2. Japanese Committee for Registration of Esophageal Carcinoma Cases. Parameters linked to ten-year survival in Japan of resected esophageal carcinoma. Chest 1989;96:1005-11.

3. Steup WH, De Leyn P, Deneffe G, Van Raemdonck D, Coosemans W, Lerut T. Tumors of the esophagogastric junction: long-term survival in relation to the pattern of lymph node metastasis and a critical analysis of the accuracy or inaccuracy of pTNM classification. J Thorac Cardiovasc Surg 1996;111:85-95.

4. Korst RJ, Rusch VW, Venkatraman E, Bains MS, Burt ME, Downey RJ, et al. Proposed revision of the staging classification for esophageal cancer. J Thorac Cardiovasc Surg 1998;115:66070

\section{Discussion}

Dr Mark J. Krasna (Baltimore, $M d$ ). The rapidly increasing incidence in esophageal cancer and the increased prevalence of Barrett's esophagus make the discussion of staging and treating esophageal cancer a highly pertinent one. This year 12,000 Americans will be diagnosed with esophageal cancer. More than 11,000 of these will be dead within 2 years, primarily from distant metastatic disease. If we could identify subgroups of patients preoperatively, we could allocate treatment options where the greatest benefit would result with the least risk.

This retrospective review of 140 patients from The Cleveland Clinic identified 36 patients with M1a disease, that is, metastasis to nonregional lymph nodes. Survival was only 5 months even in those with M1a disease. Clearly, treatment philosophy changed during this trial. Why, for instance, did 7 patients with distant metastatic disease undergo surgical resection?

The authors point out that clinical staging was not done, and the manuscript points out that the authors did not include those patients whose malignant disease was downstaged with chemotherapy or radiation therapy. Although Steup and associates, from Belgium, reported a 17\% 5-year survival in this subset of patients, the current report showed poor overall survival. Our report by Ellis and colleagues showed a $28 \%$ to $38 \%$ 5-year survival with small lesions despite positive celiac lymph nodes. Although the Walsh study showed poor survival with surgery alone, the recent intergroup study showed a reasonable survival with surgery alone, including some patients who also had thoracic esophageal lesions with positive celiac nodes.

Rather than taking a nihilistic approach to this problem, we would suggest the following algorithm. Nowadays celiac or thoracic lymph nodes can easily be assessed with the aid of modern staging techniques. We have shown a 94\% accuracy using laparoscopic lymph node staging in the past. Recently, Reed and associates have published excellent staging results with EUS-guided needle biopsies of celiac lymph nodes. If lymph nodes are found to be positive, patients should be enrolled in a study to see whether aggressive chemotherapy and radiation therapy with or without surgery may offer any advantage for survival. If results of such studies are negative, these patients should be offered palliative care, whereas other patients should be treated aggressively. In a recent phase II study of 48 patients subjected to surgical staging and trimodality therapy, we reported a $66 \%$ complete pathologic response in patients with negative celiac nodes and a $20 \%$ complete pathologic response in those with positive celiac nodes. Although this series is small, the implication is that even in this subset of patients we can identify patients before treatment who, with aggressive treatment, can get a significant response.

How would you treat an otherwise good-risk patient with an adenocarcinoma of the distal esophagus who has a biopsyproven positive $1.5-\mathrm{cm}$ celiac lymph node? Would you endorse a change in the current classification to allow an N2 classification, rather than M1a, for this extent of disease?

Only with new staging techniques and large cooperative treatment protocols will we effect a change in this new epidemic of esophageal cancer.

Dr Christie. Thank you very much, Dr Krasna, for your comments. To answer your first question, how to deal with a patient who is a good surgical candidate and on preoperative evaluation has evidence of celiac node metastases, M1a disease: We have 17 patients in this group who had preoperative chemoradiation therapy. In 4 of the 17 patients, the disease was downstaged from M1a to M0, and in that group of patients, 3 had a complete pathologic response and 2 had a very good clinical outcome. One patient had an incomplete pathologic response. His disease was downstaged to M0 at surgery but he ultimately died of M1b disease. This parallels the results that you described with downstaging. Although the numbers are small, this is a $20 \%$ downstaging with chemoradiation. The approach to patients in whom preoperative evaluation discloses M1a disease should include chemotherapy or chemoradiation therapy, with a consideration for the addition of surgery, but the patient should be treated as part of a study protocol.

To answer your second question regarding whether the staging should be changed to N2 disease, I do not think so. Although survival is possible, survival parallels metastatic disease to distant organs.

Dr Mark B. Orringer (Ann Arbor, Mich). This is an important issue. Our current staging system for esophageal cancer has some real deficiencies. The M1a/M1b designation is one of them, and the T0 designation is another. If you now give patients with large esophageal cancers and associated adenopathy on computed tomographic scan chemotherapy and radiation therapy, and they are complete responders with no residual cancer in the resected specimen, they are considered to have T0 disease. But similarly, patients with intramucosal carcinoma, which has one of the best prognoses, are also considered to have T0 disease. We are lumping apples and oranges into the $\mathrm{T} 0$ category, and that is not optimal.

At the meeting of the American Surgical Association, we presented our series of 1085 transhiatal resections. Eight hundred of these resections were for cancer, and we virtually have no survival at 5 years for patients with either stage IVA 
or stage IVB disease. Like Dr Christie, I think there may be a small difference in stage IVA versus stage IVB. However, for practical purposes, $90 \%$-plus of these patients will be dead within 5 years, most within 3 years. The question will come up, if you are doing an esophagectomy and you find a $1-\mathrm{cm}$ nodule in the liver, shouldn't you proceed with the esophagectomy because, after all, that poor patient has dysphagia and needs palliation? Well, that poor patient will probably be dead within 6 months, and I have trouble subjecting him or her to a major resection under those circumstances.

What is a "celiac" node? If the involved node is right at the origin of the left gastric artery from the celiac axis and it can be resected with a specimen, we tend to designate it a "left gastric node." If the node is to the right of the celiac axis and cannot be resected en bloc with the rest of the specimen, then that becomes a "celiac node." But is there really a survival difference in patients who have such "central" nodal spread?

We have repeatedly found that when the nodes at the origin of the left gastric artery near the celiac trunk are involved with tumor, the survival is dismal. I guess I am in Mark Krasna's corner here. If you are the practicing surgeon and you know preoperatively that the patient has celiac node disease, you should not be doing an esophageal resection unless that patient has had some sort of a protocol attempt to eliminate that adenopathy to provide better survival. Otherwise, you are doing a palliative operation. Unless you can do it with a mortality that is in the low single digits and get the patient out of the hospital within a week, you have done more harm than good.

Dr Antoon E. M. R. Lerut (Leuven, Belgium). I would like to congratulate you on this study because it emphasizes a very difficult problem.

Reasonable survival can be obtained with primary surgery. Dr Krasna mentioned 17\%. In our more recent experience, with 2-field and 3-field extended lymphadenectomies, survival is more than $20 \%$ in advanced stage carcinoma. What we learned from the operation is that indeed we are dealing with a very bad clinical staging system. When you are doing this kind of operation and you do a 3-field lymphadenectomy, you will find $30 \%$ positive lymph nodes in the neck, and that is where the problem arises. In that sort of situation we have a mean of up to 9 positive lymph nodes, and that is the point at which our clinical staging is failing, including the minimally invasive thoracoscopic or laparoscopic staging. Also, the echoendoscopists are failing. They tell me that when the patient is in a flat position, a particular lymph node is seen on the celiac axis, but when the patient is on the left side, the same lymph node will be on the gastric artery. That makes a difference between the N1 and M1a classifications. That is another reason that we have so much difficulty in appreciating the results of induction chemotherapy. We simply do not know what we are talking about because we do not have the correct stage. Perhaps the positron emission tomographic scan will help us in the future. However, my suggestion is that there is indeed a place for an N2 classification rather than M1. In this respect we have to measure tumor load, that is, the number of positive lymph nodes, as was proposed 20 years ago by Skinner. If there are fewer than 4 positive nodes (N1), then primary surgery should yield a good 5-year survival. If there are more positive lymph nodes (N2), then you probably are failing, and I think that is the lesson.

Dr Christie. We agree with that. Certainly there is evidence that the number of lymph nodes may have a very important effect on survival. The purpose of our study was to address the relevance of the new change of the staging system.

Dr Carolyn E. Reed (Charleston, SC). I may be a bit confused. I think this is a terribly important discussion because adenocarcinoma of the distal esophagus and gastroesophageal junction is becoming increasingly prevalent. As you know, metastasis to the left gastric/celiac axis is very common. One of your coauthors, Dr Rice, has shown that most of us see T3 tumors. With T3 tumors, there is more than $75 \%$ nodal involvement, and with distal adenocarcinomas, the nodal involvement will be along the lesser curvature or the celiac axis.

Are you saying that if you have an adenocarcinoma of the gastroesophageal junction at The Cleveland Clinic and if EUS suggests that a celiac node is enlarged, you administer chemotherapy and radiation therapy but no longer consider the patient to be a surgical candidate? I also echo what other people have said, that we need to try to prove up front whether these are positive nodes before we make these judgments. However, sometimes it is very difficult to say whether a node is a proximal left gastric lymph node or a true celiac axis lymph node. There are a few centimeters that can make the difference. What is your new policy at The Cleveland Clinic?

Dr Christie. Dr Reed, I am no longer at The Cleveland Clinic. Dr Rice may want to elaborate.

Dr Rice. I would be happy to elaborate. We use EUS to stage the disease in our patients. If the patient has large celiac axis nodes, we are now using fine-needle aspiration. If that patient has M1a disease demonstrated by fine-needle aspiration, the prospect for survival is dismal. Surgery alone is not offered to that patient. Instead, induction chemoradiation therapy is offered with a resection on protocol. 